\title{
PAPEL DO FORAME OVAL PATENTE E DA VÁLVULA DE EUSTÁQUIO NOS EVENTOS TROMBOEMBÓLICOS
}

\author{
Elza Magalhães ${ }^{1,2}$, Jorge Torreão ${ }^{3}$, Nila Costa ${ }^{2}$, Nelmacy Freitas ${ }^{4}$, Ailton Melo ${ }^{1}$ \\ RESUMO - A associação entre forame oval patente (FOP) e embolia paradoxal é assunto já descrito na \\ literatura médica. Quando não existe associação com aneurisma de septo atrial, surgem dúvidas quanto à \\ origem do trombo devido à dificuldade que se tem de documentar a passagem de êmbolos vindos da \\ circulação venosa pelo shunt direita-esquerch. Descrevemos quatro casos de eventos vasculares, cujos \\ ecoca rdiogramas transesofágicos demonstraram a presença de trombo aderido ao FOP associado à válvula \\ de Eustáquio proeminente na ausência de aneurisma de septo. Esse artigo visa ilustrar e chamar atenção \\ para o potencial papel do FOP e da válvula de Eustáquio na ocorrência da embolia paradoxal
}

PALAVRAS-CHAVES: forame oval patente, válvula de Eustáquio, embolia paradoxal.

\begin{abstract}
Role of patent foramen ovale and of the Eustaquian valve in the trhomboembolic events
ABSTRACT- The association between patent foramen ovale (PFO) and paradoxical emboli is described in medical literature. When have not association with atrial septal aneurysm, arguments arise about the clots origin, because it have been difficult to gave evidence of the clots passing from venous circulation across shunt right to left. We describe four cases of vascular events whose transesophageal echocardiography showed clots nailed within the PFO associated the prominent Eustachian valve, without septal aneurysm. This paper aim to illustrate and to warm about the potentialy role of the PFO and Eustachian valve in the paradoxical embolism.
\end{abstract}

KEY WORDS: patent foramen ovale, Eustachian valve, paradoxical emboli.

O forame oval é uma estrutura embriológica indispensável à sobrevivência do feto. Ele é formado pela superposição das porções livres dos septos pri mum e secundum, pe rmitindo a existência de um orifício virtual necessário à passagem de sangue do lado direito para o esquerdo do coração ${ }^{1,3}$. Após o nascimento, ele já não se faz mais necessário, por isso o forame de fecha. Em cerca de $27 \%$ da população adulta o forame persiste aberto devido a uma fusão inadequada dos septos primum e secundum².

A válvula de Eustáquio é também uma estrutura importante na manutenção da circulação fetal. Ela é proeminente nessa fase da vida, e tem a função de direcionar o fluxo proveniente da veia cava inferior para a circulação sistêmica através do forame oval patente FOP. Após o nascimento, com o fechamento do forame oval, a válvula de Eustáquio regride, tornando-se fenestrada, sendo então chamada rede de Chiari $^{4}$ (Fig 1). Uma outra ocorrência também encontrada em alguns adultos é uma válvula de Eustáquio proeminente (Fig 1B) que parece ter importância na ocorrência de embolia paradoxal quando se associa ao FOP, facilitando o direcionamento de fluxo do átrio direito para o esquerdo e, consequentemente, a passagem de coágulos provenientes da circulação venosa ${ }^{4}$.

Em 1877, um patologista alemão, Cohnhein, durante necropsia de uma mulher jovem que morreu de acidente vascular cerebral (AVC), descreveu a presença de forame oval e levantou a hipótese de que a passagem de êmbolos, através dele, tivesse sido a causa do evento. Essa foi a primeira descrição de embolização paradoxal da literatura ${ }^{4}$. Posteriormente, outros trabalhos descreveram a associação entre embolia paradoxal e defeitos de septo atrial5,6. Os dados da literatura são mais consistentes quando se trata de embolia paradoxal em pacientes com FOP associada a aneurisma de septo. No que diz respeito à FOP, isoladamente, ainda existem discussões ${ }^{4,5}$.

Não raramente, nos deparamos com pacientes ví-

\footnotetext{
'Divisão de Neurologia e Epidemiologia da Universidade Federal da Bahia, Salvador BA, Brasil; ${ }^{2}$ Hospital Português - Beneficência P o rtuguesa do Estado da Bahia, Salvador BA, Brasil; ${ }^{3}$ Santa Casa de Misericórdia da Bahia - Hospital Santa Isabel, Salvador BA, Brasil; ${ }^{4}$ Hospital São Rafael, Salvador BA, Brasil.
}

Recebido 14 Junho 2005, recebido na forma final 11 Outubro 2005. Aceito 10 Novembro 2005. 


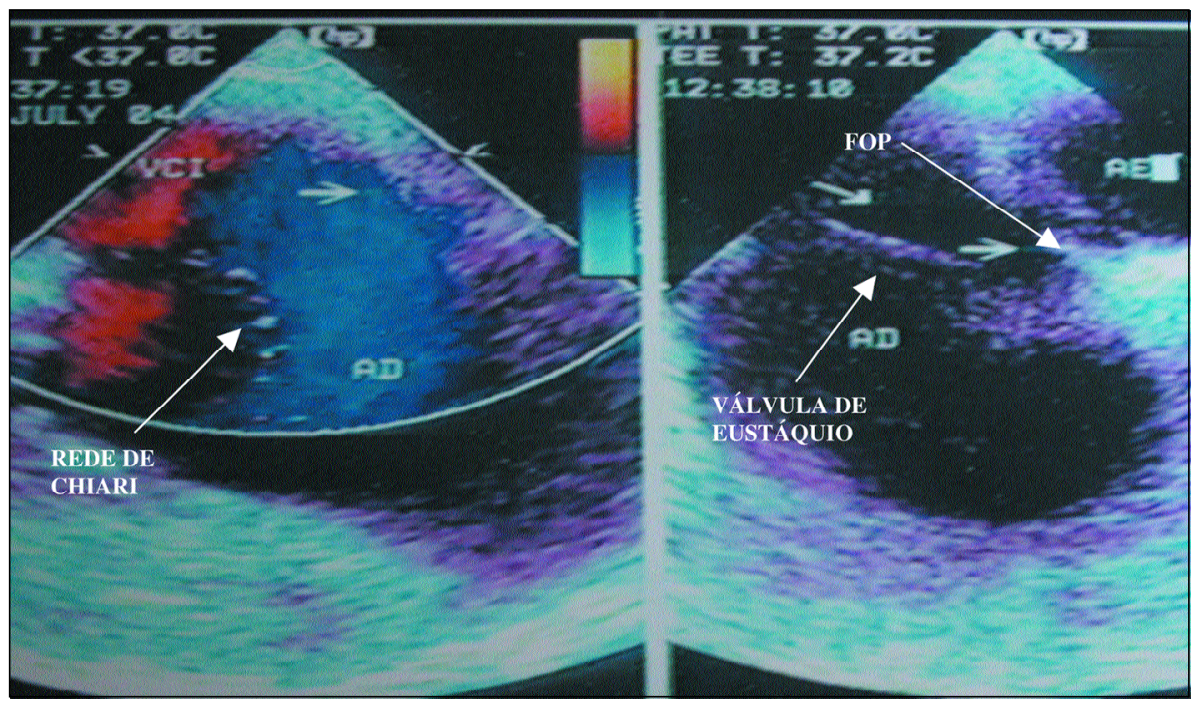

Fig 1. Imagens reveladas durante ETE de um dos casos relatados mostrando a rede de Chiari e a válvula de Eustáquio direcionando fluxo para o FOP.

timas de AVC em que não é possível definir a etiologia. Em muitos deles encontramos apenas FOP como possível causa, porém muitos desses diagnósticos são ditos como criptogênicos devido à dificuldade de se documentar a fonte emboligênica ${ }^{5}$. Em alguns pacientes com embolia paradoxal não se encontra trombose venosa em membros inferiores, embora esse achado não afaste a possibilidade de ser essa a fonte de êmbolos. Outro mecanismo aventado para embolia associado a FOP é que o trombo seja formado no próprio forame devido à estase de sangue pela baixa diferença de pressão entre os átrios em algumas fases do ciclo cardíaco ${ }^{5,6}$. O método diagnóstico de escolha para confirmação de FOP é o ecocardiograma transesofágico (ETE). A alta sensibilidade desse método permite obter imagens cardíacas de regiões não acessíveis pela via transtorácica e, através do recurso de Doppler colorido com contraste salino, é possível documentar a presença de shunt da D-E2,7. Um outro método diagnóstico que confirma a passagem de sangue através do forame é o Doppler transcraniano ${ }^{8}$. Esse recurso permite a visualização de microbolhas na circulação cerebral provenientes do coração após a injeção de solução salina por via venosa ${ }^{8}$. Recentemente, vem sendo discutida qual a melhor conduta terapêutica para portadores de FOP com eventos cerebrovasculares. Alguns autores sugerem o fechamento do forame por cateterismo com colocação de próteses ${ }^{9,10}$. O u tros propõem o uso de antiagregantes plaquetários, justificado porque o uso de anticoagulantes orais leva a eventos hemorrágicos e que ainda não existe definição quanto ao risco benefício para colocação de próteses via cateterismo ${ }^{3,4}$.
Relatamos quatro casos de pacientes com diagnóstico de eventos vasculares, sendo dois com AVC. Os ETE mostraram, em todos eles, a presença de trombo aderido ao FOP além de uma válvula de Eustáquio proeminente. Nenhum tinha aneurisma de septo atrial. Nosso objetivo é reforçar a teoria de que o FOP tem papel importante no risco de eventos vasculares mesmo quando não está associado ao aneurisma de septo. A proeminência da válvula de Eustáquio talvez tenha contribuído para esses eventos.

\section{CASOS}

Caso 1 - Mulher de 36 anos de idade, atendida com quadro de disartria, hemiparesia esquerda e sonolência. Tomografia computadorizada de crânio mostrou imagem hipodensa no território da artéria cerebral média direita compatível com AVC isquêmico agudo (Fig 2A). Duplex scan confirmou trombose venosa profunda. ETE revelou aumento das câmeras cardíacas direitas com pressão sistólica na artéria pulmonar (PSAP) elevada $(45 \mathrm{mmHg})$ e um trombo aderido ao FOP (Fig 2B). Encaminhada para ciru rgia de urgência tendo sido retirado o trombo e corrigido o shunt.

Caso 2 - Mulher de 45 anos de idade. Estava no quarto dia após revascularização miocárdica quando apresentou quadrode embolia pulmonar. O ETE mostrou aumento de câmeras cardíacas direitas com disfunção sistólica do ventrículo direito, PSAP de $100 \mathrm{~m} \mathrm{mHg}$ e um trombo aderido ao FOP (Fig 3A). Optou-se pela heparinização devido à contra-indicação para ciru rgia e para trombolítico. ETE de controle não mostrou mais a presença de tombo.

Caso 3 - Homem de 71 anos de idade, portador de prótese biológica em posição mitral há oito anos, em ritmo de fibrilação atrial crônica. Admitido com dor abdominal, dispnéia e hemoptise devido a embolia pulmonar grave. 


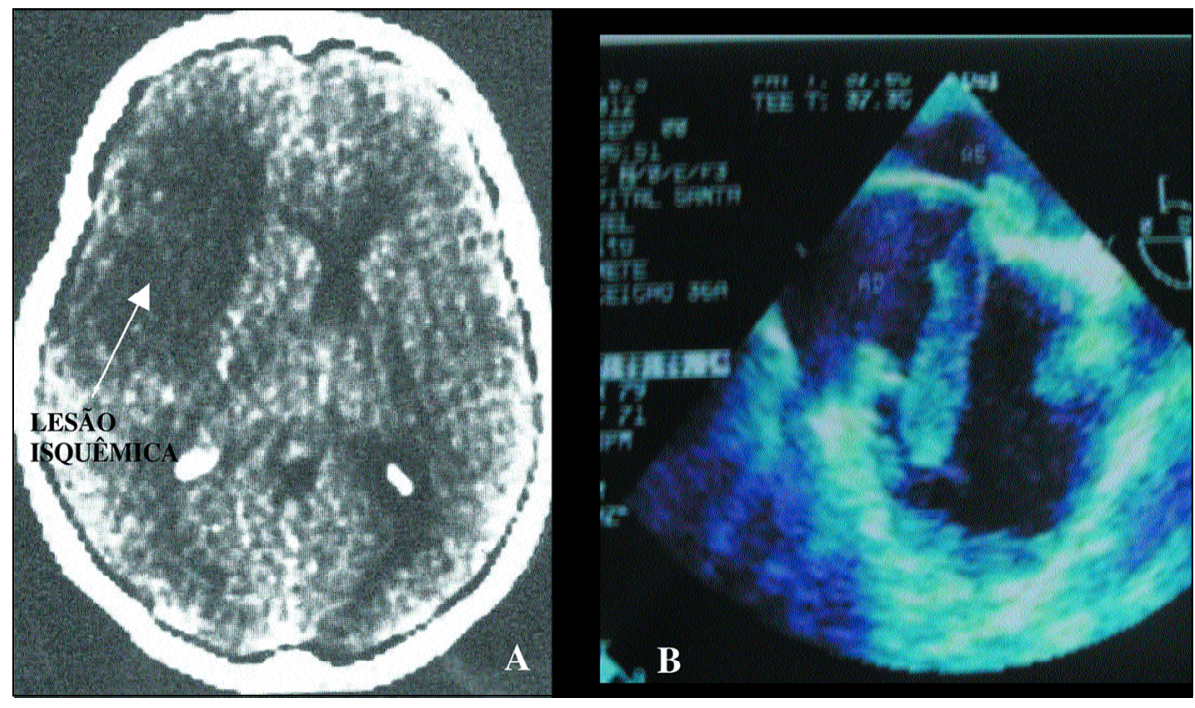

Fig 2. A) Tomografia computadori zada de crânio do paciente (caso 1) mostrando lesão isquêmica no terri tório da artéria cerebral média direi ta. B) Imagem revelada durante o ETE desse mesmo paciente mostran do tombo aderido ao FOP.
Fig 3. A) Foto do ecocardiograma do paciente (caso 2) mostrando trombo aderido ao FOP. B) Imagem revelada durante o ecocardiogra ma do paciente (caso 3) mostrando trombo aderido ao FOP.

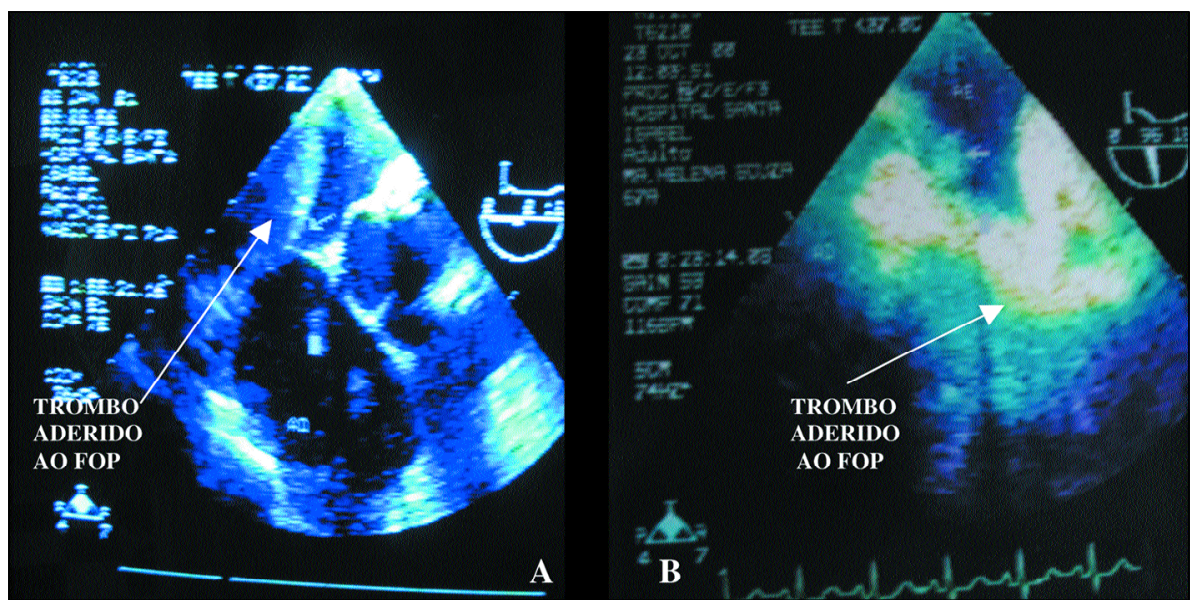

Fig 4. A) Ressonância magnética de crânio do paciente (caso 4) mos trando extensa lesão isquêmica em te ritório da artéria cerebral média esquerda. B) Foto revelada durante ETE do mesmo paciente mostrando um tombo aderido ao FOP. 
ETE mostrou trombo aderido ao FOP com porção móvel no átrio direito (Fig $3 \mathrm{~B}$ ). O paciente evoluiu para o óbito antes da terapêutica adequada.

Caso 4 - Homem de 47 anos de idade, atendido com sonolência, afasia, hemiplegia direita. Não foram identificados fatores de risco para doença cérebro vascular. Ressonância magnética de crânio revelou imagem isquêmica extensa no território da artéria cerebral média esquerda (Fig 4A) e o ETE demonstrou grande trombo no FOP com porção móvel em ambos os átrios (Fig 4B ). O paciente evoluiu para o óbito.

\section{DISCUSSÃO}

Estudos prévios têm mostrado associação entre FOP e eventos tromboembólicos. Embora existam sugestões de que essa associação possa ser casual, alguns autores relacionam um maior risco de AVC com o tamanho e grau do shunt além de aceitar que associação com aneurisma de septo aumentou esse ris$\mathrm{CO}^{4,5,9}$. Ainda não existe consenso a respeito do papel do FOP isolado nos eventos cerebrovasculares. A Academia Americana de Neurologia publicou, em abril de 2004, uma revisão sistemática com objetivo de estabelecer condutas para pacientes com AVC e FOP com e sem aneurisma de septo ${ }^{4}$. Seus autores concluíram que FOP isoladamente não aumenta o risco de recorrência de AVC e que a melhor opção terapêutica para esses pacientes ainda não está estabelecida ${ }^{4}$. A concomitância de aneurisma de septo com FOP aumenta o risco para eventos embólicos ${ }^{4,5}$. Continua ainda em discussão qual o mecanismo pelo qual o FOP sozinho causa embolia.

A passagem de trombos vindos da circulação venosa pelo FOP é o mecanismo mais aceito para explicar eventos vasculares em portadores de FOP sem outros fatores de risco. Existem sugestões de que o trombo também possa ser formado no próprio forame, nas fases do ciclo cardíaco em que a diferença de pressão entre os átrios diminui ${ }^{6}$. Os casos aqui relatados apresentam aspectos que podem ser explicados por ambas as teorias.

O caso 1 apresenta trombose venosa em membro inferior, sendo a embolia paradoxal o mecanismo mais provável para o evento. $O$ trombo visto no caso 2 pode ter migrado do sistema venoso profundo ou sido formado no próprio coração. Ambos os mecanismos podem ter sido facilitados pela imobilização do pós-operatório. O paciente 3 era portador de fibrilação atrial, causa importante de trombo intracardíaco. O último caso, com AVC sem outros fatores de risco identificados, não teve a fonte emboligênica definida.

Um achado comum entre os casos relatados, além da presença de trombo aderido ao FOP, foi a ausência de aneurisma de septo e a presença de uma válvula de Eustáquio proeminente. Isso fortalece a teoria de que não é necessária a associação de FOP com aneurisma de septo para que ocorram eventos embólicos como sugere a literatura ${ }^{4}$. A válvula de Estáquio proeminente parece ter alguma participação nesse processo, talvez por direcionar o fluxo sanguíneo para o forame oval, facilitando assim a passagem de êmbolos.

Apesar dos debates acerca de qual o risco de ocorrência de eventos vasculares nos port a d o res de FOP, a sua participação foi documentada aqui, tanto isoladamente quanto associada a outros fatores como fibrilação atrial e trombose venosa em membros inferiores. A presença da válvula de Eustáquio talvez mereça mais atenção em pacientes com FOP. Estudos controlados para definir qual a melhor conduta em portadores de FOP com eventos vasculares são necessários.

\section{REFERÊNCIAS}

1. Mas JL, A rquizan C, Lamy C, et al. Recurrent cerebrovascular events associated with patent foramen ovale, atrial septal aneurysm, or both. N Engl J Med 2001;345:1740-1746.

2. Brickner EL, Hillis LD, Lange RA. Congenital heart disease in adults. N Engl J Med 2000;27:256-263.

3. Méier B, Lock JE. Contemporary management of patent foramen ovale. Circulation 2003;107:5-9.

4. Messé SR, Silverman IE, Kizer JR, et al. Practice parameter: recurrent stroke with patent foramen ovale and atrial septal aneurysm. Report of the Quality Standards Subcommittee of the American Academy of Neurology. Neurology 2004;62:1042-1050.

5. Lamy C, Giannesini C, Zuber M, et al. Clinical and imaging findings in cryptogenic stroke patients with and without patent foramen ovale: The PFO-ASA study. Atrial septal aneurysm. Stroke 2002;33:706-711.

6. Horton SC, Bunch TJ. Patent foramen ovale and stroke. Mayo Clin Proc 2004;79:79-88.

7. Daniel WG, Mugge A. Transesophageal echocardiography. N Engl J Med 1995;332:1268-1279.

8. Anzola GP. Clinical impact of patent foramen ovale diagnosis with transcranial doppler. Eur J Ultrasound 2002;16:11-20.

9. CastroDS, Cartoni D, Fiorelli M, et al. Morphological and functional characteristics of patent forâmen ovale and their embolic implications. Stoke 2000;31:2407-2413.

10. LandzbergMJ, Khairy P. Indications for the closure of patent foramen ovale. Heart 2004;90:219-224 